\title{
Critical behaviour of confined systems
}

\author{
A.V.Chalyi ${ }^{1}$, K.A.Chalyi ${ }^{2}$, L.M.Chernenko ${ }^{3}$, A.N.Vasil'ev ${ }^{4}$ \\ 1 Department of Physics, National Medical University, \\ 13 Shevchenko Blvd., Kiev 01601, Ukraine \\ 2 Dept. Bio. and Chem. Engineering, Gunma University, \\ Kiryu 376-8515, Japan \\ 3 Institute of Surface Chemistry \\ of the National Academy of Sciences of Ukraine, \\ 31 Nauki Avenue, Kiev 252028, Ukraine \\ 4 Department of Physics, Kiev University, \\ 6 Glushkova Avenue, Kiev 03022, Ukraine
}

Received February 23, 2000

\begin{abstract}
The critical phenomena and peculiarities of phase transitions in the confined fluid systems are investigated. A system with the geometry of a planeparallel layer is chosen in order to discuss the influence of the space limitations on the critical characteristics of fluids. The main ideas of the Munster iteration procedure were used to find the pair and the direct correlation functions. Such an important characteristic of the system as the correlation length was found and correspondent results were analyzed in the terms of the scaling theory. Special attention is paid to the calculation of the shifts of the critical parameters (critical temperature and density). The three-moment approximation is used to investigate anisotropic liquids. The system of the Ornstein-Zernike (OZ) integral equations is involved to investigate the correlative properties of the binary fluid mixtures. It is shown for the fluids with the isomorphic character of the interaction that the approximation may be used that makes the system similar to the $\mathrm{OZ}$ onecomponent liquid model.

The asymptotic formulae for the pair correlation functions are found and the validity of the Munster method for the binary mixtures is considered. The peculiarities of critical light opalescence for the systems with the special geometry are considered.
\end{abstract}

Key words: critical phenomena, phase transitions, confined system, correlation function

PACS: $05.70 . F h, 05.70 . J k$

\section{Introduction}

A number of achievements of the modern theory of critical phenomena and phase transitions are connected with the application of powerful methods of statistical 
physics, namely the method of collective variables by I.R.Yukhnovskii and his collaborators [1].

The space limitation is one of the main factors determining the critical behaviour of the fluid systems undergoing phase transitions. In general case the whole set of the main results for the critical region may be received using only the pair correlation function of the order parameter fluctuations of the system. So it is crucial to get the information about the pair correlation function for the finite-size systems in order to use it hereafter. As it is well known, the pair correlation function of the density fluctuations may be expressed in the case of the space infinite one-component liquid system with a scaling formula [2]:

$$
G_{2}(r)=A \exp \left(-r / R_{\mathrm{c}}\right) / r^{(1+\eta)}
$$

where $\eta \approx 0.034$ is the critical exponent and $R_{\mathrm{c}}$ is the correlation length. Due to the long-ranged correlation at the critical point the correlation length demonstrates an anomalous growth. For many practical and theoretical purposes the OZ model of free fluctuation field [3] could be used. In the framework of this model it is possible to find the asymptotic formulae for the correlation functions and the corresponding correlation length and calculate the shifts of the critical parameters. Namely, for the abovementioned system the zeroth approximation for the pair correlation function is equivalent to the (1.1) when $\eta=0$ :

$$
G_{2}(r)=A \exp \left(-r / R_{\mathrm{c}}\right) / r
$$

Unfortunately, this asymptotic expression has a singularity when its argument is approaching to zero. Therefore, it would be better to solve this problem using the Munster method. The main idea of this method is an application of the integral and differential OZ equations (or systems of OZ equations in the case of binary mixtures system) and corresponding iteration procedure in order to obtain new correct expressions for the correlation functions [4]. It is important to stress that even after the first step of iteration process the singularity [5] of the pair correlation function disappears.

Let us consider the one-component liquid system. The OZ integral equation has the following form $[3,6,7]$ :

$$
G_{2}\left(\mathbf{r}_{1}, \mathbf{r}_{2}\right)=f\left(\mathbf{r}_{1}, \mathbf{r}_{2}\right)+\int \rho\left(\mathbf{r}^{\prime}\right) f\left(\mathbf{r}_{1}, \mathbf{r}^{\prime}\right) G_{2}\left(\mathbf{r}^{\prime}, \mathbf{r}_{2}\right) \mathrm{d} \mathbf{r}^{\prime}
$$

As a rule the direct correlation function $f\left(\mathbf{r}_{1}, \mathbf{r}^{\prime}\right)$ differs significantly from zero only when the distance $\left|\mathbf{r}_{1}-\mathbf{r}^{\prime}\right|$ between the interacting particles is small. So in the case of an isotropic system when $f\left(\mathbf{r}_{1}, \mathbf{r}^{\prime}\right)=f\left(\left|\mathbf{r}_{1}-\mathbf{r}^{\prime}\right|\right)$ and $G_{2}\left(\mathbf{r}_{1}, \mathbf{r}^{\prime}\right)=G_{2}\left(\left|\mathbf{r}_{1}-\mathbf{r}^{\prime}\right|\right)$ one can transform the equation (1.3) using the Taylor expansion for the direct correlation function to the differential OZ equation [4]:

$$
\left(\nabla^{2}-\kappa^{2}\right) G_{2}(\mathbf{r})=-\frac{f(\mathbf{r})}{C_{2}}
$$


Here, the so-called two-moment approximation was used with two spatial moments of the direct correlation function $C_{0}=\int \rho(\mathbf{r}) f(\mathbf{r}) \mathrm{d} \mathbf{r}, C_{2}=\frac{1}{6} \int \rho(\mathbf{r}) f(\mathbf{r}) \mathbf{r}^{2} \mathrm{~d} \mathbf{r}$, and $\kappa^{2}=$ $\left(1-C_{0}\right) / C_{2}, \rho(\mathbf{r})$ is the local density. When the system is considerably anisotropic one must take into account the first spatial moment of the direct correlation function. For this situation the differential OZ equation is

$$
\begin{aligned}
& \sum_{i, j=1}^{3} a_{i j}\left(\mathbf{r}_{2}\right) \frac{\partial^{2} G_{2}\left(\mathbf{r}_{1}, \mathbf{r}_{2}\right)}{\partial x_{1 i} \partial x_{1 j}}-\vec{C}_{1} \cdot \vec{\nabla}_{1} G_{2}\left(\mathbf{r}_{1}, \mathbf{r}_{2}\right)-\left(1-C_{0}\right) G_{2}\left(\mathbf{r}_{1}, \mathbf{r}_{2}\right)= \\
& \quad=-f\left(\mathbf{r}_{1}, \mathbf{r}_{2}\right)
\end{aligned}
$$

where the following definitions were used: $\vec{C}_{1}\left(\mathbf{r}_{2}\right)=\int \rho(\mathbf{r}) f\left(\mathbf{r}, \mathbf{r}_{2}\right) \mathbf{r} \mathrm{d} \mathbf{r}, a_{i j}\left(\mathbf{r}_{2}\right)=$ $a_{j i}\left(\mathbf{r}_{2}\right)=\frac{1}{2} \int \rho\left(x_{1}, x_{2}, x_{3}\right) f\left(x_{1}, x_{2}, x_{3} \mid \mathbf{r}_{2}\right) x_{i} x_{j} \mathrm{~d} x_{1} \mathrm{~d} x_{2} \mathrm{~d} x_{3}$ and $C_{2}=\frac{1}{3}\left(a_{11}+a_{22}+a_{33}\right)$. Lets assume that $a_{i i}(\mathbf{r}) \cong C_{2}=$ const, $\vec{C}_{1}(\mathbf{r}) \equiv \vec{C}_{1}$ and $\rho(\mathbf{r})=\langle\rho\rangle=$ const. Then we can get from $(1.5)$

$$
\triangle G_{2}+G_{2 x y} \frac{a_{12}}{C_{2}}+G_{2 x z} \frac{a_{13}}{C_{2}}+G_{2 y z} \frac{a_{23}}{C_{2}}-\vec{a} \vec{\nabla} G_{2}-\kappa^{2} G_{2}=-\frac{f}{C_{2}},
$$

where vector $\vec{a}=\vec{C}_{1} / C_{2}$. This equation may be simplified for the systems with a special symmetry.

\section{Plane-parallel layer system}

Let us consider the system with the geometry of a plane-parallel layer of the thickness $2 h$. Such systems have a significant practical importance because many real physical and biological systems are spatially finite-sized and their geometrical form may be taken as a plane-parallel [8].

\subsection{The correlation functions}

Taking the zeroth boundary conditions to satisfy the limiting transition to the infinite system one may find the direct and the pair correlation functions in the following form $[8,9]$ :

$$
G_{2}(\rho, z)=\sum_{m=0}^{\infty} G_{2(m)}(\rho) \cos (\pi(m+0.5) z / h)
$$

and

$$
f(\rho, z)=\sum_{m=0}^{\infty} f_{(m)}(\rho) \cos (\pi(m+0.5) z / h) .
$$

Using the zeroth approximation for the direct correlation function $f^{(0)}(\mathbf{r})=C_{0} \delta(\mathbf{r}) /$ $\langle\rho\rangle$ and taking into account that

$$
\delta(z)=\frac{1}{h} \sum_{m=0}^{\infty} \cos \left(\frac{\pi(2 m+1) z}{2 h}\right)
$$


one has the following equations for the harmonics of correlation functions from the integral (1.3) and differential (1.4) OZ equations:

$$
G_{2(m)}(\rho)=f_{(m)}(\rho)+h\langle\rho\rangle \int f_{(m)}\left(\rho_{1}\right) G_{2(m)}\left(\left|\vec{\rho}-\overrightarrow{\rho_{1}}\right|\right) \mathrm{d} \overrightarrow{\rho_{1}}
$$

and

$$
\left(\nabla^{2}-\kappa_{m}^{2}\right) G_{2(m)}(\rho)=-\frac{C_{0} \delta(\rho)}{\langle\rho\rangle h C_{2}}
$$

where $\kappa_{m}^{2}=\kappa^{2}+\pi^{2}(2 m+1)^{2} / 4 h^{2}$. These equations may be used for the calculation of the correlation functions by the iteration procedure. So from the (2.5) one can find the asymptotic formula for the pair correlation function $[5,8,9]$ :

$$
G_{2}^{(0)}(\rho, z)=\frac{C_{0}}{2 \pi h\langle\rho\rangle C_{2}} \sum_{m=0}^{\infty} K_{0}\left(\rho \kappa_{m}\right) \cos (\pi(2 m+1) / 2 h) .
$$

Using then the integral OZ equation (2.4) it is possible to calculate the first approximation for the direct correlation function [5]:

$$
f^{(1)}(\rho, z)=\frac{C_{0}}{2 \pi h\langle\rho\rangle C_{2}} \sum_{m=0}^{\infty} K_{0}\left(\rho \sqrt{\kappa_{m}^{2}+C_{0} / C_{2}}\right) \cos (\pi(2 m+1) / 2 h) .
$$

Then one may find the first iteration for the pair correlation function [5] from equation (2.5):

$$
G_{2}^{(1)}(\rho, z)=\frac{1}{2 \pi h\langle\rho\rangle C_{2}} \sum_{m=0}^{\infty}\left[K_{0}\left(\rho \kappa_{m}\right)-K_{0}\left(\rho \tilde{\kappa}_{m}\right)\right] \cos (\pi(2 m+1) / 2 h),
$$

where $\tilde{\kappa}_{m}=\sqrt{\kappa_{m}^{2}+C_{0} / C_{2}}$. As it was stressed above the first iteration of the pair correlation function has no singularities and is more realistic than the asymptotic expression.

\subsection{The correlation length}

It is natural to determine the correlation length as the normalized second spatial moment of the pair correlation function $[8,9]$ :

$$
R_{\mathrm{c}}=\sqrt{\frac{\int G_{2}(\mathbf{r}) \mathbf{r}^{2} \mathrm{~d} \mathbf{r}}{\int G_{2}(\mathbf{r}) \mathrm{d} \mathbf{r}}}
$$

From (2.10) one can find, after integrating and leaving only the first harmonics in the (2.8), the following expression for the correlation length:

$$
R_{\mathrm{c}}^{2}=R_{x y}^{2}+R_{z}^{2},
$$

where

$$
R_{x y}^{2}=\frac{4}{\kappa^{2}+\frac{\pi^{2}}{4 h^{2}}}+\frac{4}{\kappa^{2}+\frac{\pi^{2}}{4 h^{2}}+\frac{C_{0}}{C_{2}}}
$$


and

$$
R_{z}^{2}=\left(1-\frac{8}{\pi^{2}}\right) h^{2} .
$$

As it is seen from the $(2.10)-(2.12)$ at the bulk critical point $(\kappa=0)$ the correlation length has a finite value. Thus the space limitation causes the shifts of the critical parameters $[5,8]$.

\subsection{The shifts of the critical parameters}

According to the scaling-law theory, parameter $\kappa$ may be presented in the following form [2]:

$$
\kappa=\tau^{\nu} f_{1}\left(\triangle \rho / \tau^{\beta}\right)=\triangle \rho^{\zeta} f_{2}\left(\tau / \triangle \rho^{1 / \beta}\right)
$$

Here $\tau=\left(T-T_{\mathrm{c}}\right) / T_{\mathrm{c}}$ and $\triangle \rho=\left(\rho-\rho_{\mathrm{c}}\right) / \rho_{\mathrm{c}}$ are the deviations of the temperature and density from their critical values respectively, $\nu=0.625, \beta=0.325$ and $\zeta=1.923$ are the critical exponents. The asymptotic behaviour of a scaling functions $f_{1}(u)$ and $f_{2}(u)$ are as follows:

$$
f_{1}(u \rightarrow 0)=\kappa_{0}=\text { const }, \quad f_{1}(u \rightarrow \infty) \sim u^{\zeta}
$$

and

$$
f_{2}(u \rightarrow 0)=\kappa_{0}=\text { const }, \quad f_{2}(u \rightarrow \infty) \sim u^{\nu}
$$

Thus using the expressions (2.10)-(2.12) for the correlation length and dependence of the parameter $\kappa$ on the temperature and density one can find the shifts of the critical parameters with respect to its values at the bulk critical point. The new critical temperature is

$$
T_{\mathrm{c}}^{*}=\frac{T_{\mathrm{c}}}{1+\left(\kappa_{0} h \Delta\right)^{-1 / \nu}}
$$

and the new critical density is

$$
\rho_{\mathrm{c}}^{*}=\frac{\rho_{\mathrm{c}}}{1+\left(\kappa_{0} h \Delta\right)^{-1 / \zeta}}
$$

where $\Delta=\sqrt{1+8 / \pi^{2}+16 /\left(\pi^{2}+4 h^{2} C_{0} / C_{2}\right)}$. These results are in good agreement with the experimental data by Lutz [10].

\section{Anisotropic systems}

In many cases the real systems are essentially anisotropic and it leads to some considerable effects. Let us consider such systems. 


\subsection{The spatially nonfinite system}

In the case of a spatially infinite system the asymptotic formula for the pair correlation function of the order parameter fluctuations [11] can be found from the equation (1.6):

$$
\begin{aligned}
G_{2}^{(0)}(\mathbf{r}) & =\frac{C_{0}}{4 \pi\langle\rho\rangle C_{2}} \cdot \frac{\exp \left(\operatorname{arcos} \theta / 2-r \sqrt{\kappa^{2}+a^{2} / 4}\right)}{r} \\
& =\frac{C_{0}}{4 \pi\langle\rho\rangle C_{2}} \cdot \frac{\exp \left(a z / 2-r \sqrt{\kappa^{2}+a^{2} / 4}\right)}{r} .
\end{aligned}
$$

Here the direction of the z-axis coincides with the direction of the vector $\vec{a}$ and $\theta$ is the angle between the vectors $\vec{r}$ and $\vec{a}$. The following iterations may be found from the integral OZ equation (1.3). The first iteration for the direct correlation function is

$$
\begin{aligned}
f^{(1)}(\mathbf{r}) & =\frac{C_{0}}{4 \pi\langle\rho\rangle C_{2}} \cdot \frac{\exp \left(\operatorname{arcos} \theta / 2-r \sqrt{\kappa^{2}+a^{2} / 4+C_{0} / C_{2}}\right)}{r} \\
& =\frac{C_{0}}{4 \pi\langle\rho\rangle C_{2}} \cdot \frac{\exp \left(a z / 2-r \sqrt{\kappa^{2}+a^{2} / 4+C_{0} / C_{2}}\right)}{r}
\end{aligned}
$$

and the first iteration for the pair correlation function is

$$
\begin{aligned}
G_{2}^{(1)}(\mathbf{r})= & \frac{1}{4 \pi\langle\rho\rangle C_{2}} \cdot \frac{\exp \left(\operatorname{arcos} \theta / 2-r \sqrt{\kappa^{2}+a^{2} / 4}\right)}{r} \\
& -\frac{1}{4 \pi\langle\rho\rangle C_{2}} \cdot \frac{\exp \left(\operatorname{arcos} \theta / 2-r \sqrt{\kappa^{2}+a^{2} / 4+C_{0} / C_{2}}\right)}{r} \\
= & \frac{1}{4 \pi\langle\rho\rangle C_{2}} \cdot \frac{\exp \left(a z / 2-r \sqrt{\kappa^{2}+a^{2} / 4}\right)}{r} \\
& -\frac{1}{4 \pi\langle\rho\rangle C_{2}} \cdot \frac{\exp \left(a z / 2-r \sqrt{\kappa^{2}+a^{2} / 4+C_{0} / C_{2}}\right)}{r} .
\end{aligned}
$$

The last formula presents the dependence of the correlative properties of the system on the angle $\theta$ and may be used to investigate the correlation length and shifts of the critical parameters [11].

\subsection{The correlation length}

Let's find the correlation length of the spatially infinite anisotropic system. Taking into account (2.13) and from (3.1) one has the following formula [11]:

$$
\begin{aligned}
R_{\mathrm{c}}(\tau) & =\frac{1}{\sqrt{\kappa^{2}+a^{2} / 4}-a \cos \theta / 2} \\
& =\frac{R_{0}}{\sqrt{\tau^{2 \nu}+a^{2} R_{0}^{2} / 4}-a R_{0} \cos \theta / 2} .
\end{aligned}
$$


Here $R_{0}=\kappa_{0}^{-1}$. It is clearly seen that critical temperature in this case depends on the direction and thus the anisotropy of the system causes the change in the critical parameters in comparison with the isotropic system.

\subsection{The new critical parameters}

The new critical temperature $T_{\mathrm{c}}^{*}$ may be found from the formula (3.4) for the correlation length:

$$
T_{\mathrm{c}}^{*}=\frac{T_{\mathrm{c}}}{1+\left(R_{0} a \sin ^{2}(\theta / 2)\right)^{1 / \nu}} .
$$

In the same way one may find the new critical density:

$$
\rho_{\mathrm{c}}^{*}=\frac{\rho_{\mathrm{c}}}{1+\left(R_{0} a \sin ^{2}(\theta / 2)\right)^{\beta / \nu}} .
$$

The maximal shift of the critical parameters takes place when $\theta=\pi$ and there is no shift (in comparison with isotropic system) when $\theta=0$.

\subsection{Plane-parallel layer}

Let us consider the anisotropic system with a geometry of a plane-parallel layer. In the situation when $\vec{a}=(0,0, a)$ one may find, using the (1.3) and (1.6), the asymptotic expression and the first iterations for the direct and the pair correlation functions. So the asymptotic formula for the pair correlation function is

$$
G_{2}^{(0)}(\mathbf{r})=\frac{C_{0} \exp \left(\frac{a z}{2}\right)}{2 \pi h C_{2}\langle\rho\rangle} \sum_{m=0}^{\infty} K_{0}\left(\rho \sqrt{\kappa_{m}^{2}+a^{2} / 4}\right) \cos \left(\frac{(m+0.5) z \pi}{h}\right) .
$$

The first approximation for the direct and pair correlation functions are as follows:

$$
\begin{aligned}
f^{(1)}(\mathbf{r})= & \frac{C_{0} \exp \left(\frac{a z}{2}\right)}{2 \pi h C_{2}\langle\rho\rangle} \sum_{m=0}^{\infty} K_{0}\left(\rho \sqrt{\tilde{\kappa}_{m}^{2}+a^{2} / 4}\right) \cos \left(\frac{(m+0.5) z \pi}{h}\right) \\
G_{2}^{(1)}(\mathbf{r})= & \frac{\exp \left(\frac{a z}{2}\right)}{2 \pi h C_{2}\langle\rho\rangle} \sum_{m=0}^{\infty}\left[K_{0}\left(\rho \sqrt{\kappa_{m}^{2}+a^{2} / 4}\right)-K_{0}\left(\rho \sqrt{\tilde{\kappa}_{m}^{2}+a^{2} / 4}\right)\right] \\
& \times \cos \left(\frac{(m+0.5) z \pi}{h}\right) .
\end{aligned}
$$

In the case when vector $\vec{a}$ has no z-component one may accept without any restriction of the generality that $\vec{a}=(0, a, 0)$ and get, similar to the previous situation, the asymptotic formula for the pair correlation function

$$
G_{2}^{(0)}(\mathbf{r})=\frac{C_{0} \exp \left(\frac{a y}{2}\right)}{2 \pi h C_{2}\langle\rho\rangle} \sum_{m=0}^{\infty} K_{0}\left(\rho \sqrt{\kappa_{m}^{2}+a^{2} / 4}\right) \cos \left(\frac{(m+0.5) z \pi}{h}\right) .
$$

The first approximation for the direct correlation function is

$$
f^{(1)}(\mathbf{r})=\frac{C_{0} \exp \left(\frac{a y}{2}\right)}{2 \pi h C_{2}\langle\rho\rangle} \sum_{m=0}^{\infty} K_{0}\left(\rho \sqrt{\tilde{\kappa}_{m}^{2}+a^{2} / 4}\right) \cos \left(\frac{(m+0.5) z \pi}{h}\right) .
$$


And the first approximation for the pair correlation function

$$
\begin{aligned}
G_{2}^{(1)}(\mathbf{r})= & \frac{\exp \left(\frac{a y}{2}\right)}{2 \pi h C_{2}\langle\rho\rangle} \sum_{m=0}^{\infty}\left[K_{0}\left(\rho \sqrt{\kappa_{m}^{2}+a^{2} / 4}\right)-K_{0}\left(\rho \sqrt{\tilde{\kappa}_{m}^{2}+a^{2} / 4}\right)\right] \\
& \times \cos \left(\frac{(m+0.5) z \pi}{h}\right) .
\end{aligned}
$$

When the vector $\vec{a}$ has an arbitrary direction the situation is much more complicated. In this case one may take $\vec{a}=(0, a \sin \theta, a \cos \theta)$. So the equation (1.6) transforms as follows:

$$
\Delta G_{2}+s \frac{\partial^{2} G_{2}}{\partial y \partial z}-a \sin \theta \frac{\partial G_{2}}{\partial y}-a \cdot \cos \theta \cdot \frac{\partial G_{2}}{\partial z}-\kappa^{2} G_{2}=-\frac{f}{C_{2}},
$$

where $s=a_{23} / \sqrt{a_{22} \cdot a_{33}} \approx a_{23} / C_{2}$. Making the substitution $x \rightarrow x, y \rightarrow(y-$ $(s z) / 2) / \sqrt{1-s^{2} / 4}, z \rightarrow z$ and using new variables $x, u, z$ one has the follow equations from (1.3) and (1.6)

$$
\Delta G_{2}-a \cos \theta \frac{\partial G_{2}}{\partial z}-\left[\frac{2 a \sin \theta-a s \cos \theta}{\sqrt{4-s^{2}}}\right] \frac{\partial G_{2}}{\partial u}-\kappa^{2} G_{2}=-\frac{f}{C_{2}}
$$

and

$$
\begin{aligned}
G_{2}(x, u, z)= & f(x, u, z)+\langle\rho\rangle \sqrt{1-\frac{s^{2}}{4}} \\
& \times \int f\left(x^{\prime}, u^{\prime}, z^{\prime}\right) G_{2}\left(x-x^{\prime}, u-u^{\prime}, z-z^{\prime}\right) \mathrm{d} x^{\prime} \mathrm{d} u^{\prime} \mathrm{d} z^{\prime} .
\end{aligned}
$$

The solution of this problem is as follows:

1) For the pair correlation function the asymptotic formula may be presented in such a way

$$
\begin{aligned}
G_{2}^{(0)}(\mathbf{r})= & \frac{C_{0} \cdot \exp \left[(a z(2 \cos \theta-s \cdot \sin \theta)+a y(2 \sin \theta-s \cdot \cos \theta)) /\left(4-s^{2}\right)\right]}{2 \pi h C_{2}\langle\rho\rangle \sqrt{1-s^{2} / 4}} \\
& \times \sum_{m=0}^{\infty} K_{0}\left(\sqrt{\left(x^{2}+\frac{(2 y-s z)^{2}}{4-s^{2}}\right)\left(\kappa_{m}^{2}+\frac{a^{2}(1-s \cdot \sin (2 \theta) / 2)}{4-s^{2}}\right)}\right) \\
& \times \cos \left(\frac{(m+0.5) z \pi}{h}\right) .
\end{aligned}
$$

2) The first approximation for the direct correlation function

$$
\begin{aligned}
f^{(1)}(\mathbf{r})= & \frac{C_{0} \cdot \exp \left[(a z(2 \cos \theta-s \cdot \sin \theta)+a y(2 \sin \theta-s \cdot \cos \theta)) /\left(4-s^{2}\right)\right]}{2 \pi h C_{2}\langle\rho\rangle \sqrt{1-s^{2} / 4}} \\
& \times \sum_{m=0}^{\infty} K_{0}\left(\sqrt{\left(x^{2}+\frac{(2 y-s z)^{2}}{4-s^{2}}\right)\left(\tilde{\kappa}_{m}^{2}+\frac{a^{2}(1-s \cdot \sin (2 \theta) / 2)}{4-s^{2}}\right)}\right) \\
& \times \cos \left(\frac{(m+0.5) z \pi}{h}\right) .
\end{aligned}
$$


3) The first approximation for the pair correlation function

$$
\begin{aligned}
G_{2}^{(1)}(\mathbf{r})= & \frac{\exp \left[(a z(2 \cos \theta-s \cdot \sin \theta)+a y(2 \sin \theta-s \cdot \cos \theta)) /\left(4-s^{2}\right)\right]}{2 \pi h C_{2}\langle\rho\rangle \sqrt{1-s^{2} / 4}} \\
& \times \sum_{m=0}^{\infty}\left[K_{0}\left(\sqrt{\left(x^{2}+\frac{(2 y-s z)^{2}}{4-s^{2}}\right)\left(\kappa_{m}^{2}+\frac{a^{2}(1-s \cdot \sin (2 \theta) / 2)}{4-s^{2}}\right)}\right)\right. \\
& \left.-K_{0}\left(\sqrt{\left(x^{2}+\frac{(2 y-s z)^{2}}{4-s^{2}}\right)\left(\tilde{\kappa}_{m}^{2}+\frac{a^{2}(1-s \cdot \sin (2 \theta) / 2)}{4-s^{2}}\right)}\right)\right] \\
& \times \cos \left(\frac{(m+0.5) z \pi}{h}\right)
\end{aligned}
$$

In the case when the anisotropy vector has its direction along the z-axis the correlation length may be found as follows:

$$
\begin{aligned}
\left(R_{\mathrm{c}} / h\right)^{2}= & +\frac{16}{4 \kappa^{2} h^{2}+\pi^{2}}-\frac{8}{a^{2} h^{2}+\pi^{2}} \\
& -\frac{8 a h \tanh (a h / 2)}{a^{2} h^{2}+\pi^{2}}+\frac{16 a^{2} h^{2}}{\left(a^{2} h^{2}+\pi^{2}\right)^{2}} .
\end{aligned}
$$

In the limiting transition $\rightarrow 0$ it gives the zeroth approximation of the correlation length of the isotropy system $[8,9]$.

\section{The binary fluid mixture}

The integral OZ system for the binary mixture has the following form:

$$
G_{i j}(\mathbf{r})=f_{i j}(\mathbf{r})+\sum_{k=1}^{2}\left\langle\rho_{k}\right\rangle \int G_{i k}\left(\mathbf{r}-\mathbf{r}_{1}\right) f_{k j}\left(\mathbf{r}_{1}\right) \mathrm{d} \mathbf{r}_{1},
$$

where $f_{i j}(\mathbf{r})=f_{j i}(\mathbf{r})$ and $G_{i j}(\mathbf{r})=G_{j i}(\mathbf{r})$ are the direct and the pair correlation functions of the components $i$ and $j$ respectively, $\left\langle\rho_{k}\right\rangle$ is the average density of the component $k$ and $i, j, k=1,2$. It is also possible to find the asymptotic expressions for the correlation functions from the OZ differential system:

$$
\sum_{m=1}^{2}\left[\frac{B_{m j}}{B_{i j}}\left(\triangle-\kappa_{m j}^{2}\right) G_{i m}(\mathbf{r})\right]=-\frac{f_{i j}(\mathbf{r})}{B_{i j}} .
$$

Here $A_{i j}=\left\langle\rho_{i}\right\rangle \int f_{i j}(\mathbf{r}) \mathrm{d} \mathbf{r}, B_{i j}=\left(\left\langle\rho_{i}\right\rangle / 6\right) \int f_{i j}(\mathbf{r}) \mathbf{r}^{2} \mathrm{~d} \mathbf{r}$ and $\kappa_{i j}^{2}=\kappa_{j i}^{2}=\left(\delta_{i j}-A_{i j}\right) / B_{i j}$.

\subsection{The zeroth approximation}

In order to receive the asymptotic formulae for the pair correlation functions let us take the Dirak delta-function as the zeroth approximation for the direct correlation functions. Taking in (4.2) $f_{i j}(\mathbf{r})=A_{i j} \delta(\mathbf{r}) /\left\langle\rho_{i}\right\rangle$ one may get the asymptotic formula for the pair correlation functions: 
1) For the spatially infinite system

$$
G_{i j}(\mathbf{r})=\frac{R_{i j}}{4 \pi \triangle q^{2} r}\left[\left(S_{i j}^{2}-q_{1}^{2}\right) \exp \left(-q_{1} r\right)-\left(S_{i j}^{2}-q_{2}^{2}\right) \exp \left(-q_{2} r\right)\right]
$$

2) For the system with the geometry of a plane-parallel layer

$$
G_{i j}(\rho, z)=\sum_{m=0}^{\infty} G_{i j(m)}(\rho) \cos (\pi(m+0.5) z / h)
$$

and

$$
\begin{aligned}
G_{i j(m)}=\frac{R_{i j}}{2 \pi h \triangle q^{2}} & {\left[\left(S_{i j}^{2}-q_{1}^{2}\right) K_{0}\left(\rho \sqrt{q_{1}^{2}+\frac{\pi^{2}(2 m+1)^{2}}{4 h^{2}}}\right)\right.} \\
& \left.-\left(S_{i j}^{2}-q_{2}^{2}\right) K_{0}\left(\rho \sqrt{q_{2}^{2}+\frac{\pi^{2}(2 m+1)^{2}}{4 h^{2}}}\right)\right] .
\end{aligned}
$$

Here the following definitions are used:

$$
\begin{aligned}
q_{1,2}^{2} & =\frac{\kappa_{11}^{2}+\kappa_{22}^{2}-2 \xi^{2} \kappa_{12}^{2}}{2\left(1-\xi^{2}\right)}\left[1 \pm \sqrt{\left.1-4 \frac{\left(\kappa_{11}^{2} \kappa_{22}^{2}-\xi^{2} \kappa_{12}^{4}\right)\left(1-\xi^{2}\right)}{\left(\kappa_{11}^{2}+\kappa_{22}^{2}-2 \xi^{2} \kappa_{12}^{2}\right)^{2}}\right]}\right. \\
R_{12} & =\frac{1}{1-\xi^{2}} \cdot \frac{A_{11} / B_{11}-A_{22} / B_{22}}{B_{11}-B_{22}} \cdot \frac{B_{12}}{\left\langle\rho_{1}\right\rangle} \\
& =\frac{1}{1-\xi^{2}} \cdot \frac{A_{11} / B_{11}-A_{22} / B_{22}}{B_{11}-B_{22}} \cdot \frac{B_{21}}{\left\langle\rho_{2}\right\rangle} \\
R_{11} & =\frac{1}{1-\xi^{2}} \cdot \frac{A_{11} / B_{11}+\xi^{2} \kappa_{12}^{2}}{\left\langle\rho_{1}\right\rangle} \\
R_{22} & =\frac{1}{1-\xi^{2}} \cdot \frac{A_{22} / B_{22}+\xi^{2} \kappa_{12}^{2}}{\left\langle\rho_{2}\right\rangle} \\
S_{12}^{2} & =\frac{A_{11}-A_{22}}{A_{11} B_{22}-A_{22} B_{11}} \\
S_{11}^{2} & =\frac{\left(A_{11} / B_{11}\right) \kappa_{22}^{2}+\xi^{2} \kappa_{12}^{4}}{A_{11} / B_{11}+\xi^{2} \kappa_{12}^{2}} \\
S_{22}^{2} & =\frac{\left(A_{22} / B_{22}\right) \kappa_{11}^{2}+\xi^{2} \kappa_{12}^{4}}{A_{22} / B_{22}+\xi^{2} \kappa_{12}^{2}}
\end{aligned}
$$

and $\triangle q^{2}=q_{2}^{2}-q_{1}^{2}, \xi^{2}=B_{12} B_{21} / B_{11} B_{22}$.

At the critical point, the main singular part of the correlation length is determined as follows:

1) For the infinite system

$$
R_{\mathrm{c}}^{2}=\frac{1}{q_{1}^{2}}+\frac{1}{q_{2}^{2}}=\frac{\kappa_{11}^{2}+\kappa_{22}^{2}-2 \xi^{2} \kappa_{12}^{2}}{\kappa_{11}^{2} \kappa_{22}^{2}-\xi^{2} \kappa_{12}^{4}} .
$$


2) For a plane-parallel layer

$$
R_{\mathrm{c}}^{2}=\frac{1}{q_{1}^{2}+\frac{\pi^{2}}{4 h^{2}}}+\frac{1}{q_{2}^{2}+\frac{\pi^{2}}{4 h^{2}}}
$$

The latter formula may be used to obtain the new critical temperature. It is obvious that in the case of a binary mixture system with the geometry of a planeparallel layer the values of the critical parameters depend on the thickness of the layer as it was before for the one-component system.

\subsection{The approximation of asymptotic behaviour}

Let us make the following substitution in the system (4.1): $G_{i j}=\langle\rho\rangle / \sqrt{\left\langle\rho_{i}\right\rangle\left\langle\rho_{j}\right\rangle} g_{i j}$ and $f_{i j}=\langle\rho\rangle / \sqrt{\left\langle\rho_{i}\right\rangle\left\langle\rho_{j}\right\rangle} C_{i j}$. The density $\langle\rho\rangle$ may be taken in any convenient way. The system of equations (4.1) transforms to the form as follows:

$$
g_{i j}(\mathbf{r})=C_{i j}(\mathbf{r})+\langle\rho\rangle \sum_{k=1}^{2} \int g_{i k}\left(\mathbf{r}-\mathbf{r}_{1}\right) C_{k j}\left(\mathbf{r}_{1}\right) \mathrm{d} \mathbf{r}_{1} .
$$

Taking into account the physical sense of the integrals in the (4.1) it is natural to accept that

$$
\int g_{12}\left(\mathbf{r}-\mathbf{r}_{1}\right) C_{11}\left(\mathbf{r}_{1}\right) \mathrm{d} \mathbf{r}_{1}=\int g_{11}\left(\mathbf{r}-\mathbf{r}_{1}\right) C_{12}\left(\mathbf{r}_{1}\right) \mathrm{d} \mathbf{r}_{1}
$$

and

$$
\int g_{12}\left(\mathbf{r}-\mathbf{r}_{1}\right) C_{22}\left(\mathbf{r}_{1}\right) \mathrm{d} \mathbf{r}_{1}=\int g_{22}\left(\mathbf{r}-\mathbf{r}_{1}\right) C_{12}\left(\mathbf{r}_{1}\right) \mathrm{d} \mathbf{r}_{1} .
$$

Let us take into account the function

$$
V(\mathbf{r})=\frac{\int g_{12}\left(\mathbf{r}-\mathbf{r}_{1}\right) C_{22}\left(\mathbf{r}_{1}\right) \mathrm{d} \mathbf{r}_{1}}{\int g_{11}\left(\mathbf{r}-\mathbf{r}_{1}\right) C_{12}\left(\mathbf{r}_{1}\right) \mathrm{d} \mathbf{r}_{1}}=\frac{\left\langle\rho_{2}\right\rangle \int G_{12}\left(\mathbf{r}-\mathbf{r}_{1}\right) f_{22}\left(\mathbf{r}_{1}\right) \mathrm{d} \mathbf{r}_{1}}{\left\langle\rho_{1}\right\rangle \int G_{11}\left(\mathbf{r}-\mathbf{r}_{1}\right) f_{12}\left(\mathbf{r}_{1}\right) \mathrm{d} \mathbf{r}_{1}}
$$

It is natural to suppose that the function $V(\mathbf{r})$ weakly depends on the $\mathbf{r}$ e.i. $V=$ const. In this case one has

$$
g_{22}(\mathbf{r})=V g_{11}(\mathbf{r}), \quad C_{22}(\mathbf{r})=V C_{11}(\mathbf{r})
$$

and

$$
g_{12}(\mathbf{r})=\sqrt{V} g_{11}(\mathbf{r}), \quad C_{12}(\mathbf{r})=\sqrt{V} C_{11}(\mathbf{r})
$$

Therefore, the system of the integral equations separates on the three equations similar to the $\mathrm{OZ}$ equation for one-component liquids:

$$
g_{i j}(\mathbf{r})=C_{i j}(\mathbf{r})+\left\langle\rho_{i j}\right\rangle \int C_{i j}\left(\mathbf{r}_{1}\right) \cdot g_{i j}\left(\mathbf{r}-\mathbf{r}_{1}\right) \mathrm{d} \mathbf{r}_{1},
$$

where $\left\langle\rho_{i j}\right\rangle=\langle\rho\rangle(1+V) / V^{(i+j-2) / 2}$. So one can find the pair and the direct correlation function for the binary mixture. Thus for the spatially infinite system: 
1) The asymptotic formula for the pair correlation function

$$
G_{i j}^{(0)}(\mathbf{r})=\frac{C_{0} V^{(i+j-2) / 2}}{4 \pi C_{2}(1+V) \sqrt{\left\langle\rho_{i}\right\rangle\left\langle\rho_{j}\right\rangle}} \cdot \frac{\exp (-\kappa r)}{r} .
$$

2) The first iteration for the direct correlation function

$$
f_{i j}^{(1)}(\mathbf{r})=\frac{C_{0} V^{(i+j-2) / 2}}{4 \pi C_{2}(1+V) \sqrt{\left\langle\rho_{i}\right\rangle\left\langle\rho_{j}\right\rangle}} \cdot \frac{\exp (-\tilde{\kappa} r)}{r}
$$

3) The first iteration for the pair correlation function

$$
G_{i j}^{(1)}(\mathbf{r})=\frac{V^{(i+j-2) / 2}}{4 \pi C_{2}(1+V) \sqrt{\left\langle\rho_{i}\right\rangle\left\langle\rho_{j}\right\rangle}} \cdot \frac{\exp (-\kappa r)-\exp (-\tilde{\kappa} r)}{r} .
$$

Here $C_{0}=\left\langle\rho_{1}\right\rangle \int f_{11}(\mathbf{r}) \mathrm{d} \mathbf{r}, C_{2}=(1 / 6)\left\langle\rho_{1}\right\rangle \int f_{11}(\mathbf{r}) \mathbf{r}^{2} \mathrm{~d} \mathbf{r}, \kappa^{2}=\left[1-(1+V) C_{0}\right] /(1+$ $V) C_{2}$ and $\tilde{\kappa}^{2}=\kappa^{2}+C_{0} / C_{2}$.

In the case of a plane-parallel layer one has:

1) The zeroth iteration for the pair correlation function

$$
G_{i j}^{(0)}(\mathbf{r})=\frac{C_{0} V^{(i+j-2) / 2}}{2 \pi h C_{2}(1+V) \sqrt{\left\langle\rho_{i}\right\rangle\left\langle\rho_{j}\right\rangle}} \sum_{m=0}^{\infty} K_{0}\left(\rho \kappa_{m}\right) \cos \left(\frac{\pi z(2 m+1)}{2 h}\right) .
$$

2) The first iteration for the direct correlation function

$$
f_{i j}^{(1)}(\mathbf{r})=\frac{C_{0} V^{(i+j-2) / 2}}{2 \pi h C_{2}(1+V) \sqrt{\left\langle\rho_{i}\right\rangle\left\langle\rho_{j}\right\rangle}} \sum_{m=0}^{\infty} K_{0}\left(\rho \tilde{\kappa}_{m}\right) \cos \left(\frac{\pi z(2 m+1)}{2 h}\right) .
$$

3) The first iteration for the pair correlation function

$$
\begin{aligned}
G_{i j}^{(1)}(\mathbf{r})= & \frac{V^{(i+j-2) / 2}}{2 \pi h C_{2}(1+V) \sqrt{\left\langle\rho_{i}\right\rangle\left\langle\rho_{j}\right\rangle}} \\
& \times \sum_{m=0}^{\infty}\left[K_{0}\left(\rho \kappa_{m}\right)-K_{0}\left(\rho \tilde{\kappa}_{m}\right)\right] \cos \left(\frac{\pi z(2 m+1)}{2 h}\right) .
\end{aligned}
$$

The shift of the critical temperature $\Delta T_{\mathrm{c}}$ in this case in comparison with the critical temperature $T_{\mathrm{c}}$ of the one-component liquid is determined as

$$
\Delta T_{\mathrm{c}}=\frac{T_{\mathrm{c}}}{1+\left(V R_{0}^{2} C_{0} / C_{2}\right)^{-1 / 2 \nu}}
$$

The latter formula (4.28) may be used to find the parameter $V$ from the corresponding experimental data. 


\subsection{The diagonal form of the $\mathrm{OZ}$ system}

In the matrix form the integral OZ system may be presented as follows:

$$
\hat{g}(\mathbf{r})=\hat{C}(\mathbf{r})+\langle\rho\rangle \int \hat{C}\left(\mathbf{r}_{1}\right) \hat{g}\left(\mathbf{r}-\mathbf{r}_{1}\right) \mathrm{d} \mathbf{r}_{1} .
$$

Here the following definitions are used: $\hat{g}(\mathbf{r})=\left(\begin{array}{ll}g_{11}(\mathbf{r}) & g_{12}(\mathbf{r}) \\ g_{21}(\mathbf{r}) & g_{22}(\mathbf{r})\end{array}\right)$ and $\hat{C}(\mathbf{r})=$ $\left(\begin{array}{ll}C_{11}(\mathbf{r}) & C_{12}(\mathbf{r}) \\ C_{21}(\mathbf{r}) & C_{22}(\mathbf{r})\end{array}\right)$. After the Fourier transforming one has

$$
\hat{g}(\mathbf{q})=\hat{C}(\mathbf{q})+\langle\rho\rangle \hat{C}(\mathbf{q}) \hat{g}(\mathbf{q}) .
$$

Let us consider the arbitrary matrix $\hat{S}(\mathbf{q})$ and $\hat{G}(\mathbf{q})=\hat{S}(\mathbf{q}) \hat{g}(\mathbf{q}) \hat{S}^{-1}(\mathbf{q}) \hat{F}(\mathbf{q})=$ $\hat{S}(\mathbf{q}) \hat{C}(\mathbf{q}) \hat{S}^{-1}(\mathbf{q})$. Then for the new functions $\hat{G}(\mathbf{q})$ and $\hat{F}(\mathbf{q})$ the equation $(4.30)$ has got just the same form:

$$
\hat{G}(\mathbf{q})=\hat{F}(\mathbf{q})+\langle\rho\rangle \hat{F}(\mathbf{q}) \hat{G}(\mathbf{q}) .
$$

It makes possible to find the $\hat{S}$ in such a way that matrix $\hat{G}$ will have a diagonal form. For this reason let us calculate the eigenvalues of $\hat{G}$ from the equation:

$$
\operatorname{Det}\left|g_{i j}(\mathbf{q})-\lambda(\mathbf{q}) \delta_{i j}\right|=0 \text {. }
$$

It gives

$$
\lambda_{1,2}=\frac{g_{11}(\mathbf{q})+g_{22}(\mathbf{q})}{2} \pm \sqrt{g_{12}^{2}(\mathbf{q})+\left(\frac{g_{11}(\mathbf{q})-g_{22}(\mathbf{q})}{2}\right)^{2}} .
$$

The matrix $\hat{S}$ may be taken as follows:

$$
\hat{S}(\mathbf{r})=\frac{1}{g_{12}(\mathbf{q})}\left(\begin{array}{cl}
{\left[\sqrt{1+s(\mathbf{q})^{2}}-s(\mathbf{q})\right]^{-1}} & 1 \\
1 & {\left[s(\mathbf{q})-\sqrt{1+s(\mathbf{q})^{2}}\right]^{-1}}
\end{array}\right),
$$

where

$$
s(\mathbf{q})=\frac{g_{11}(\mathbf{q})-g_{22}(\mathbf{q})}{2 g_{12}(\mathbf{q})}=\frac{C_{11}(\mathbf{q})-C_{22}(\mathbf{q})}{2 C_{12}(\mathbf{q})}
$$

Thus one has

$$
G_{1,2}(\mathbf{q})=\frac{g_{11}(\mathbf{q})+g_{22}(\mathbf{q})}{2} \pm g_{12}(\mathbf{q}) \sqrt{1+s(\mathbf{q})^{2}}
$$

and

$$
F_{1,2}(\mathbf{q})=\frac{C_{11}(\mathbf{q})+C_{22}(\mathbf{q})}{2} \pm C_{12}(\mathbf{q}) \sqrt{1+s(\mathbf{q})^{2}}
$$

The system of integral equations (4.29) splits into two integral equations similar to the OZ equation for the one-component liquid system:

$$
G_{1,2}(\mathbf{r})=F_{1,2}(\mathbf{r})+\langle\rho\rangle \int F_{1,2}\left(\mathbf{r}_{1}\right) G_{1,2}\left(\mathbf{r}-\mathbf{r}_{1}\right) \mathrm{d} \mathbf{r}_{1} .
$$

The Munster scheme may be applied to the equation (4.38) in order to calculate the consequent iterations of the correlation functions because the initial iteration for the direct correlation function is commonly used as the delta-function. 


\subsection{The Munster method for binary mixtures}

For the general case of binary systems, the Munster method is available for the investigation of the pair correlation functions. Let us consider the differential OZ system in the matrix form:

$$
\triangle \hat{g}(\mathbf{r})-\hat{C}_{2}^{-1}\left(\hat{E}-\hat{C}_{0}\right) \hat{g}(\mathbf{r})=-\hat{C}_{2}^{-1} \hat{C}(\mathbf{r}) .
$$

Here were used the definitions $\hat{C}_{0}=\langle\rho\rangle \int \hat{C}(\mathbf{r}) \mathrm{d} \mathbf{r}, \hat{C}_{0}=(1 / 6)\langle\rho\rangle \int \hat{C}(\mathbf{r}) \mathbf{r}^{2} \mathrm{~d} \mathbf{r}$ and $\hat{E}$ is the unit matrix. It allows us to find the zeroth approximation for the matrix of the pair correlation functions using the initial iteration for the direct correlation functions from $(4.39) \hat{C}^{(0)}(\mathbf{r})=\left(\hat{C}_{0} /\langle\rho\rangle\right) \delta(\mathbf{r})$. So, one has got

$$
\triangle \hat{g}(\mathbf{r})-\hat{C}_{2}^{-1}\left(\hat{E}-\hat{C}_{0}\right) \hat{g}(\mathbf{r})=-\frac{\hat{C}_{2}^{-1} \hat{C}_{0}}{\langle\rho\rangle} \delta(\mathbf{r}) .
$$

For the spatially infinite system the Fourier transformation of the zeroth approximation for the matrix of the pair correlation functions $\hat{g}(\mathbf{q})$ is

$$
\hat{g}^{(0)}(\mathbf{q})=\left[\mathbf{q}^{2} \hat{E}+\hat{C}_{2}^{-1}\left(\hat{E}-\hat{C}_{0}\right)\right]^{-1} \frac{\hat{C}_{2}^{-1} \hat{C}_{0}}{\langle\rho\rangle} .
$$

The first iteration for the direct correlation functions may be found using the equation (4.30). It gives

$$
\hat{C}^{(1)}(\mathbf{q})=\left[\mathbf{q}^{2} \hat{E}+\hat{C}_{2}^{-1}\left(\hat{E}-\hat{C}_{0}\right)+\hat{C}_{2}^{-1} \hat{C}_{0}\right]^{-1} \frac{\hat{C}_{2}^{-1} \hat{C}_{0}}{\langle\rho\rangle} .
$$

It is important to stress that for conserving the initial symmetry of the integral system (4.29) the following equation has to take place:

$$
\hat{C}_{2} \hat{C}_{0}=\hat{C}_{0} \hat{C}_{2}
$$

Then from the (4.39) one can get the first iteration for the pair correlation functions

$$
\begin{aligned}
\hat{g}^{(1)}(\mathbf{q})= & {\left[\mathbf{q}^{2} \hat{E}+\hat{C}_{2}^{-1}\left(\hat{E}-\hat{C}_{0}\right)\right]^{-1} \frac{\hat{C}_{2}^{-1}}{\langle\rho\rangle} } \\
& -\left[\mathbf{q}^{2} \hat{E}+\hat{C}_{2}^{-1}\left(\hat{E}-\hat{C}_{0}\right)+\hat{C}_{2}^{-1} \hat{C}_{0}\right]^{-1} \frac{\hat{C}_{2}^{-1}}{\langle\rho\rangle} .
\end{aligned}
$$

Then, considering the system with the geometry of a plane-parallel layer, one can calculate the correlation functions in the following form:

$$
\hat{g}(\rho, z)=\sum_{m=0}^{\infty} \hat{g}_{(m)}(\rho) \cos (\pi(m+0.5) z / h)
$$

and

$$
\hat{C}(\rho, z)=\sum_{m=0}^{\infty} \hat{C}_{(m)}(\rho) \cos (\pi(m+0.5) z / h) .
$$


The integral OZ equation (4.30) gives

$$
\hat{G}_{m}(\mathbf{q})=\hat{f}_{m}(\mathbf{q})+h\langle\rho\rangle \hat{f}_{m}(\mathbf{q}) \hat{G}_{m}(\mathbf{q}) .
$$

And differential equation for the harmonics

$$
\triangle \hat{g}_{(m)}(\rho)-\left[\hat{C}_{2}^{-1}\left(\hat{E}-\hat{C}_{0}\right)+\frac{\pi^{2}(2 m+1)^{2}}{4 h^{2}} \hat{E}\right] \hat{g}_{(m)}(\rho)=-\frac{\hat{C}_{2}^{-1} \hat{C}_{0}}{h\langle\rho\rangle} \cdot \delta(\rho)
$$

Thus, using the analogy with the previous situation, one can find:

1) The zeroth approximation for the matrix of the pair correlation functions harmonics

$$
\hat{g}_{(m)}^{(0)}(\mathbf{q})=\left[\mathbf{q}^{2} \hat{E}+\frac{\pi^{2}(2 m+1)^{2}}{4 h^{2}} \hat{E}+\hat{C}_{2}^{-1}\left(\hat{E}-\hat{C}_{0}\right)\right]^{-1} \frac{\hat{C}_{2}^{-1} \hat{C}_{0}}{h\langle\rho\rangle} .
$$

2) The first approximation for the matrix of the direct correlation functions harmonics

$$
\hat{C}_{(m)}^{(1)}(\mathbf{q})=\left[\mathbf{q}^{2} \hat{E}+\frac{\pi^{2}(2 m+1)^{2}}{4 h^{2}} \hat{E}+\hat{C}_{2}^{-1}\left(\hat{E}-\hat{C}_{0}\right)+\hat{C}_{2}^{-1} \hat{C}_{0}\right]^{-1} \frac{\hat{C}_{2}^{-1} \hat{C}_{0}}{h\langle\rho\rangle}
$$

3) The first approximation for the matrix of the pair correlation functions harmonics

$$
\begin{aligned}
\hat{g}_{(m)}^{(1)}(\mathbf{q})= & {\left[\mathbf{q}^{2} \hat{E}+\frac{\pi^{2}(2 m+1)^{2}}{4 h^{2}} \hat{E}+\hat{C}_{2}^{-1}\left(\hat{E}-\hat{C}_{0}\right)\right]^{-1} \frac{\hat{C}_{2}^{-1}}{h\langle\rho\rangle} } \\
& -\left[\mathbf{q}^{2} \hat{E}+\frac{\pi^{2}(2 m+1)^{2}}{4 h^{2}} \hat{E}+\hat{C}_{2}^{-1}\left(\hat{E}-\hat{C}_{0}\right)+\hat{C}_{2}^{-1} \hat{C}_{0}\right]^{-1} \frac{\hat{C}_{2}^{-1}}{h\langle\rho\rangle}
\end{aligned}
$$

Comparing the equations (4.41), (4.42), (4.44) and (4.49), (4.50), (4.51) respectively, it is possible to make the following conclusion: the consequent iterations for the correlation functions could be expressed by the asymptotic formulae for the pair correlation functions. Just the same result was received for the one-component systems [11].

\section{Critical parameters and spatial insufficiency}

Let us consider a one-component liquid, which is filled in a cylindrical sample of the radius $a$ that extends infinitely along the z-axes, i.e. $0<x, y \leqslant a,-\infty<z<\infty$. Reduced geometry of the system leads to the change of critical characteristics while the cylinder radius became smaller. Thus the declination of the critical temperature $T_{\mathrm{c}}^{*}$, density $\rho_{\mathrm{c}}^{*}$ and viscosity $\eta^{*}$ for finite-size near-critical liquid system takes place. The geometric factor set the characteristic of spatial limitation and defined as $K=$ $a / R_{\mathrm{co}}$, where $R_{\mathrm{co}}$ is the amplitude of the correlation length. The new values of critical parameters may be defined at the point of the correlation length maximum. 


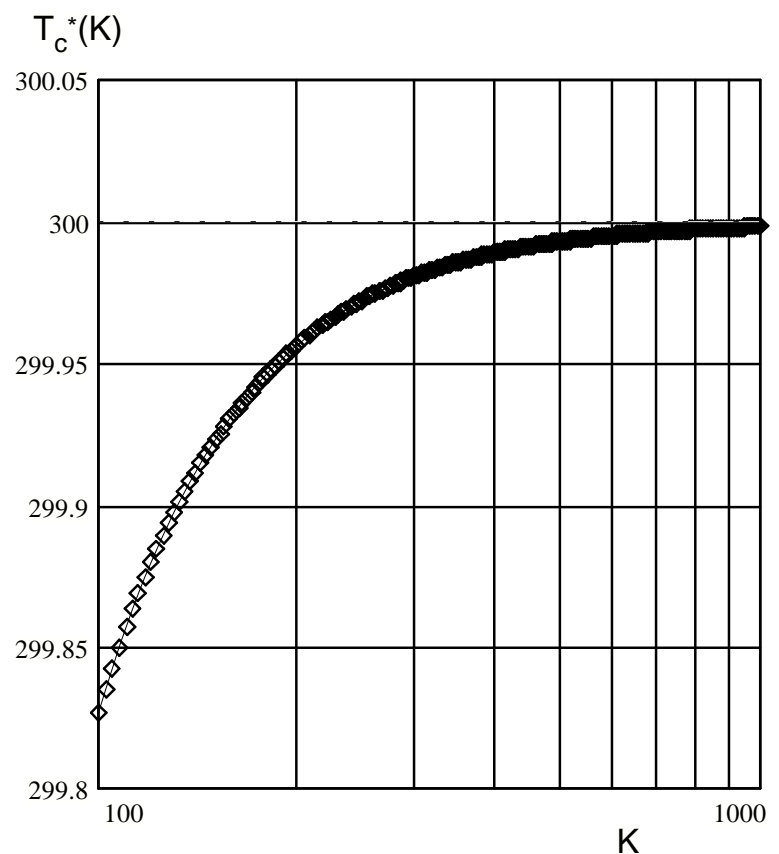

Figure 1. Dependence of the new critical temperature $T_{\mathrm{c}}^{*}(K)$ on geometrical factor $K$. Here, the assumed critical temperature for bulk system $T_{\mathrm{c}}=300 \mathrm{~K}$, critical exponent $\nu=0.5$.

To determine such an important characteristic of a liquid as viscosity the scaling formula for the correlation length of finite-size cylindrical system has to be used. Correlation length $R_{\mathrm{c}}$ in a spatially limited system of cylindrical geometry appears dependent not only on thermodynamic variables (temperature, density, etc.), but also on the geometrical factor $K$, having the sense of molecular layers number, which is possible to arrange along the radius of the cylinder. The longitudinal component of the correlation length $\left(R_{\mathrm{c}}\right)_{z}$ along the cylinder axes allows us to determine the new critical temperature $T_{\mathrm{c}}^{*}(K)$. For a sufficiently close vicinity of critical isochore where $\Delta \rho \ll \tau^{\beta}, \rho \approx \rho_{\mathrm{c}}$ and $T>T_{\mathrm{c}}$ one can get the following formula for the critical temperature of liquid in the small volume with a cylindrical geometry:

$$
T_{\mathrm{c}}^{*}(K)=T_{\mathrm{c}}\left[1+\left(\psi_{1} / K\right)^{1 / \nu}\right]^{-1} .
$$

From formula (5.1), it naturally follows that under transferring to the spatially unlimited system when the radius of the cylinder aspires to infinity $a \rightarrow \infty$ accompanied by geometrical factor $K \rightarrow \infty$, a new critical temperature $T_{\mathrm{c}}^{*}(K)$ becomes equal to the bulk critical temperature $T_{\mathrm{c}}$, i.e. there is no shift. Figure 1 illustrates the dependence of a new critical temperature $T_{\mathrm{c}}^{*}(K)$ on the geometric factor $\mathrm{K}$ for a cylindrical sample with a homogeneous (zeroth) boundary condition of the first type with the mean-field value of exponent $\nu=0.5$ and the assumed bulk critical temperature $T_{\mathrm{c}}=300 \mathrm{~K}$. The analysis of formula (5.1) shows that the shift of critical temperature for the cylindrical sample $T_{\mathrm{c}}^{*}(K)$ in comparison with the critical temperature $T_{\mathrm{c}}$ of volumetric phase may be very considerable. For example, in the case of geometric factor $K=100$ difference of critical temperature is 


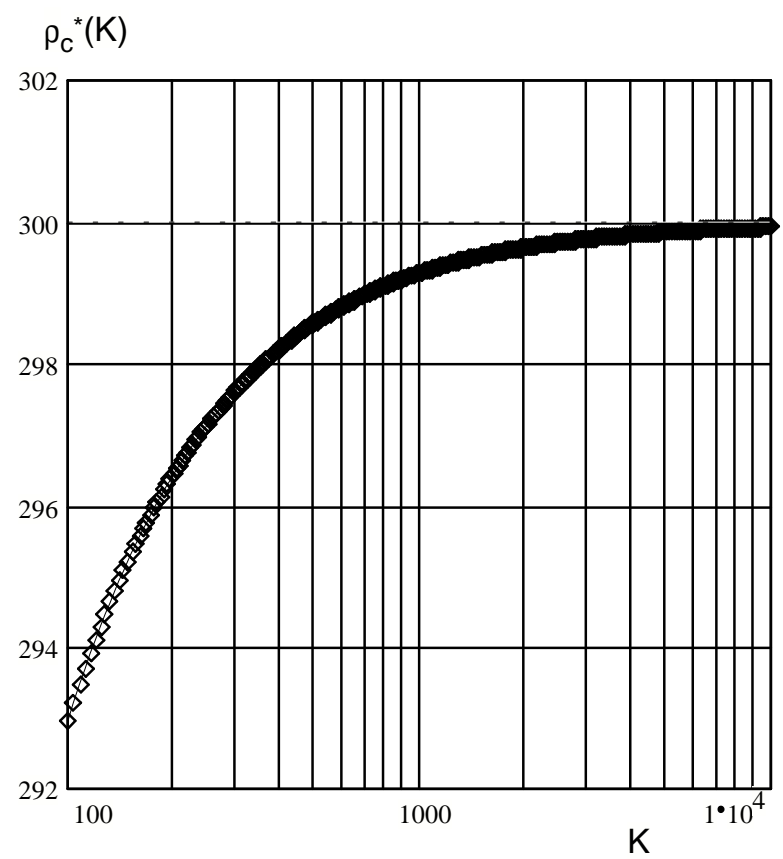

Figure 2. Dependence of the new critical density $\rho_{\mathrm{c}}^{*}(K)$ on geometrical factor $K$. Here, the assumed critical density for bulk system $\rho_{\mathrm{c}}=300 \mathrm{~kg} / \mathrm{m}^{3}$, critical exponent $\delta=3$.

$\Delta T_{\mathrm{c}}=T_{\mathrm{c}}-T_{\mathrm{c}}^{*}(K)=0.173 \mathrm{~K}$. It corresponds to the shift of critical point on $\Delta_{\tau}=6.4 \cdot 10^{-5}$ lower than bulk location.

Using just the same way as for the temperature, one can consider the changing of a new critical density $\rho_{\mathrm{c}}^{*}(K)$ in a spatially limited system of cylindrical geometry contrary to the value of critical density $\rho_{\mathrm{c}}$ for an unlimited volumetric phase. Then in the vicinity of critical isotherm, where $\Delta \rho \gg \tau^{\beta}$, for single-component liquid, the new value of critical density becomes equal to:

$$
\rho_{\mathrm{c}}^{*}(K)=\rho_{\mathrm{c}}\left[1+\left(\psi_{1} / K\right)^{2 /(\delta-1)}\right]^{-1} .
$$

From formula (5.2) follows that under transferring to the spatially unlimited system $(K \rightarrow \infty), \rho_{\mathrm{c}}^{*}(K) \rightarrow \rho_{\mathrm{c}}$ the shift of density is absent. Figure 2 illustrates the dependence of the new critical density $\rho_{\mathrm{c}}^{*}(K)$ on the geometric factor $K$ under a mean-field value of the critical exponent $\delta=3$ and $\rho_{\mathrm{c}}=300 \mathrm{~kg} / \mathrm{m}^{3}$. The analysis of formula (5.2) shows that the shift of the critical density for a cylindrical sample might be very significant. So, in the case of geometric factor $K=1000$ and $\beta=0.5$ $(2 /(\delta-1)=\beta / \nu)$ the shift of the critical density is $\rho_{\mathrm{c}}-\rho_{\mathrm{c}}^{*}(K)=0.72 \mathrm{~kg} / \mathrm{m}^{3}$. It means that the shift of the critical point on $\Delta_{\rho}=2 \cdot 10^{-3}$ lower than the bulk location. Using the above results, one could investigate the change of viscosity $\eta$. Combining the scaling relation for viscosity in the absence of shear

$$
\eta=\eta^{\mathrm{B}}\left(Q_{0} R_{\mathrm{c}}\right)^{\chi}
$$

and formula for the correlation length, the equation for viscosity $\eta^{*}$ of a spatially- 


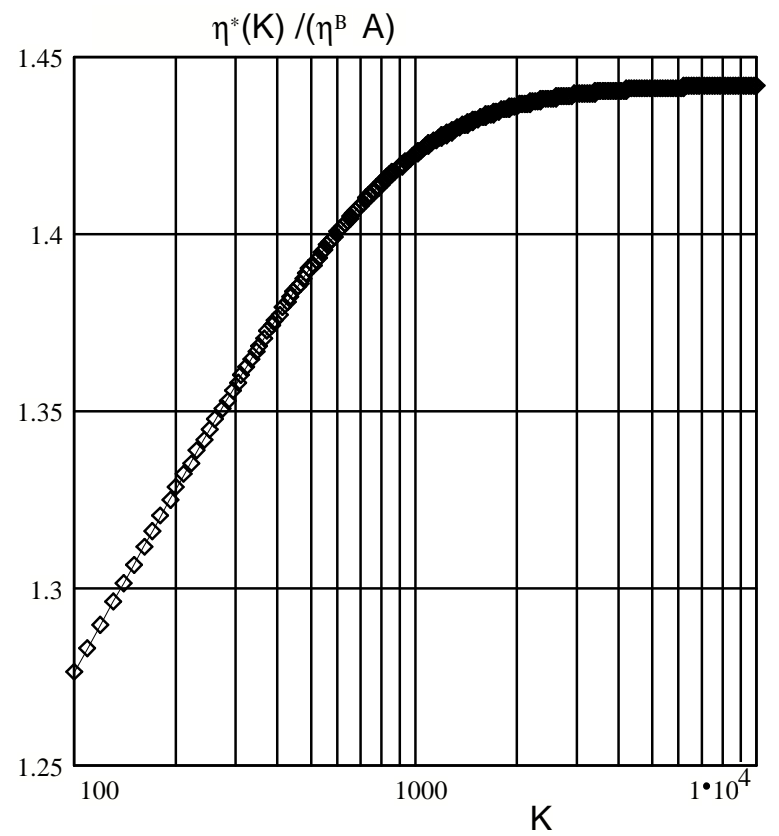

Figure 3. Dependence of the dimentionless viscosity $\eta^{*}(K) /\left(\eta^{\mathrm{B}} A\right)$ on geometrical factor $K$. Here, the assumed critical exponent $\nu=0.5$ and $\chi=0.06$, temperature declination $\tau=10^{-5}$.

limited liquid cylindrical system in near-critical state may be received

$$
\eta^{*} \sim \eta^{\mathrm{B}} A K^{\chi}\left(K^{2} \tau^{2 \nu}+\mu_{1}^{2}\right)^{-\chi / 2}
$$

where $\eta^{\mathrm{B}}$ is a background viscosity, $\chi=0.06$ is the critical exponent and $A=$ $\left(Q_{o} R_{\mathrm{co}}\right)^{\chi}$ is the system-dependent constant. As it is seen from (5.4), the viscosity in spatially limited system of cylindrical geometry depends not only on thermodynamic variable but also on the geometric factor $K$. In comparison with the spatially infinite system, for which according to the formula

$$
\eta / \eta^{\mathrm{B}} \sim \tau^{-\phi}
$$

viscosity increases at the critical temperature point $\left(T \rightarrow T_{\mathrm{c}}, \tau \rightarrow 0\right)$ up to infinity ( $\phi=0.41$ is the critical exponent), for the cylindrical sample of radius $a$ when $\tau \rightarrow 0$ the maximum value of viscosity is realized:

$$
\eta^{*}(\tau=0) \sim 0.95 a^{\chi} \eta^{\mathrm{B}} Q_{0}^{\chi}
$$

Limiting transition to a case of a spatially unlimited system at $K \rightarrow \infty$ has to be realized. From the formula (5.4) it could be seen, that this transition takes place, i.e. $\eta \sim \tau^{-\chi}$ for $K \rightarrow \infty$. Figure 3 demonstrates the dependence of viscosity $\eta^{*}$ on the geometric factor $K$ at temperature deviation $\tau=10^{-5}$. It is possible to make a conclusion that viscosity became smaller when reducing the size of the system. Figure 4 illustrates temperature dependence of $\eta^{*}$ at $K=300$. Calculations show 


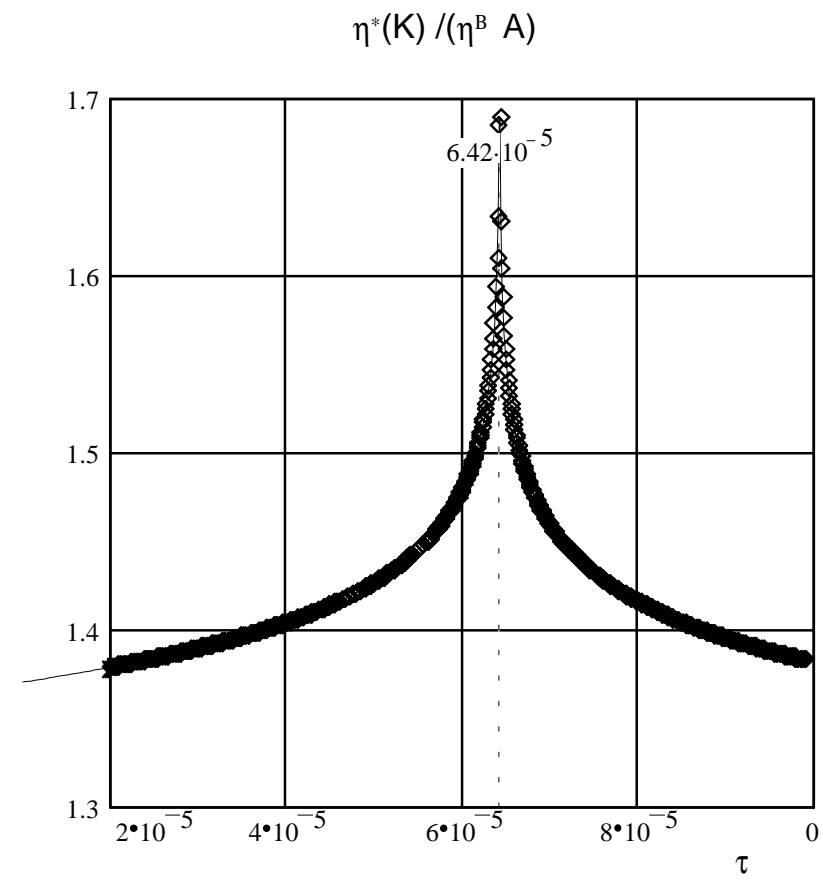

Figure 4. Temperature dependence of the dimentionless viscosity $\eta^{*}(K) /\left(\eta^{\mathrm{B}} A\right)$. Here assumed critical exponent $\nu=0.5$ and $\chi=0.06$, geometrical factor $K=300$.

that the anomalous growth of viscosity appears not at the bulk critical temperature $T=T_{\mathrm{c}}(\tau=0)$ but, as it was to be expected, at the new critical temperature $T_{\mathrm{c}}^{*}(K)$, which is the same as determined from formula (5.1). This fact gives an opportunity to define a new critical temperature in one of the two ways - from maximum of correlation length or from maximum of viscosity.

\section{Light scattering}

The study of light scattering, as it is well known, is a very fruitful method in molecular physics. Using the existing theoretical approaches and precision experimental data, it is possible to receive a reliable information about various equilibrium and kinetic properties of liquids in a broad interval of thermodynamic parameters modification including the critical region.

The description of propagation of the electromagnetic waves (in particular light) in near-critical liquids, is based on the well known conclusions, which are obtained within the framework of the main concepts of the critical light opalescence theory. To understand the specific features of the light scattering it is important to take into account the concept of spatially unlocal fluctuations in the proximity of the critical point that was suggested for the first time in the theory of OZ critical opalescence. The main point of this idea is the significance of the correlation length of fluctuations. From the standpoint of modern theories of phase transitions and critical phenomena, the OZ model actually corresponds to a model of free (noninteracting) 
fluctuations and consequently cannot give an adequate account of interactions of the order parameters, which are strongly fluctuating in immediate proximity to the critical point. However, the modern experiments prove that for the light scattering in liquids that are close to the critical point the deviations of the data from predictions of the OZ theory are minor. In many experimental and theoretical studies of critical light opalescence, the approximation of single scattering is used. The traditional description of critical opalescence in an approximation of single scattering is based on the known bound between the integral light intensity $I_{1}$ and the Fourier-image of conjugate correlation $G_{2}(\mathbf{q})$.

Of special significance are the studies of critical light opalescence in individual liquids and liquid mixtures conducted under the natural conditions of the acting external gravitational field, which results in a sharp redistribution of physical properties of a liquid depending on height. Strictly speaking, critical phenomena isomorphic to phase transitions of the second type in such liquid systems in the presence of gravitational field happen in a rather narrow transitional stratum (inter-phase). The linear size of this transitional stratum appears to be about a correlation length. This important circumstance allows us to assert that the critical phenomena in liquids in conditions of the acting gravitational field (the so-called "gravitational effect") happen, as a matter of fact, in spatially limited systems.

Let us proceed immediately to the research of dispersing properties of a spatially limited liquid system near the point of phase transition and consider a spatially limited system, which has the form of a plane-parallel stratum $(-\infty<x, y<\infty$, $-h \leqslant z \leqslant h)$. So, the description of light scattering near the critical point in the approximation of single scattering is based on the following expression:

$$
I_{1} \sim \operatorname{Re} \iint G_{2}(\rho, z) \exp \left[-\mathrm{i}\left(k_{x y} \rho+k_{z}\right)\right] \rho \mathrm{d} \rho \mathrm{d} z,
$$

where $\rho=\sqrt{x^{2}+y^{2}}$. One must take into account the main contribution in $G_{2}$ for the plane-parallel stratum, which is set by the formula $G_{2}(\rho, z)=K_{0}\left(\rho \sqrt{\kappa^{2}+\pi^{2} / 4 h^{2}}\right) \times$ $\cos (\pi z / 2 h)$, where $\kappa=R_{\mathrm{c}}^{-1}=R_{\mathrm{c} 0}^{-1} \tau^{\nu}$ is the inverse significance of a correlation length of spatially unlimited medium, $R_{\mathrm{c} 0}^{-1}=\kappa_{0}$ is the amplitude of correlation length, $K_{0}$ is the McDonald's function, $\tau=\left(T-T_{\mathrm{c}}\right) / T_{\mathrm{c}}$. Then executing an integration of expression (6.1) one receives

$$
I_{1}\left(\tau, \theta_{x y}, \theta_{z}\right) \sim \frac{(\pi / h) \cos \left(k_{z} h\right)}{\left(\kappa^{2}+\pi^{2} / 4 h^{2}+k_{x y}^{2}\right)\left(\pi^{2} / 4 h^{2}+k_{z}^{2}\right)},
$$

where $k_{x y}=(4 \pi / \lambda) \sin \left(\theta_{x y} / 2\right), k_{z}=(4 \pi / \lambda) \sin \left(\theta_{z} / 2\right)$ is the components of wave vector modification during scattering. By setting the components of the correlation length in a plane of the stratum $\left(R_{\mathrm{c}}\right)_{x y}=1 / \sqrt{\kappa^{2}+\pi^{2} / 4 h^{2}}$ and in a perpendicular direction, i.e. along the axes $\mathrm{Oz},\left(R_{\mathrm{c}}\right)_{z}=2 h / \pi$, it is possible to transform the formula (6.2) to the following form:

$$
I_{1}\left(\tau, \theta_{x y}, \theta_{z}\right) \sim \frac{(4 h) \cos \left(k_{z} h\right)}{\left(\kappa^{2}+\pi^{2} / 4 h^{2}\right)\left(1+\left(R_{\mathrm{c}}\right)_{x y}^{2} k_{x y}^{2}\right)\left(1+\left(R_{\mathrm{c}}\right)_{z}^{2} k_{z}^{2}\right)} .
$$




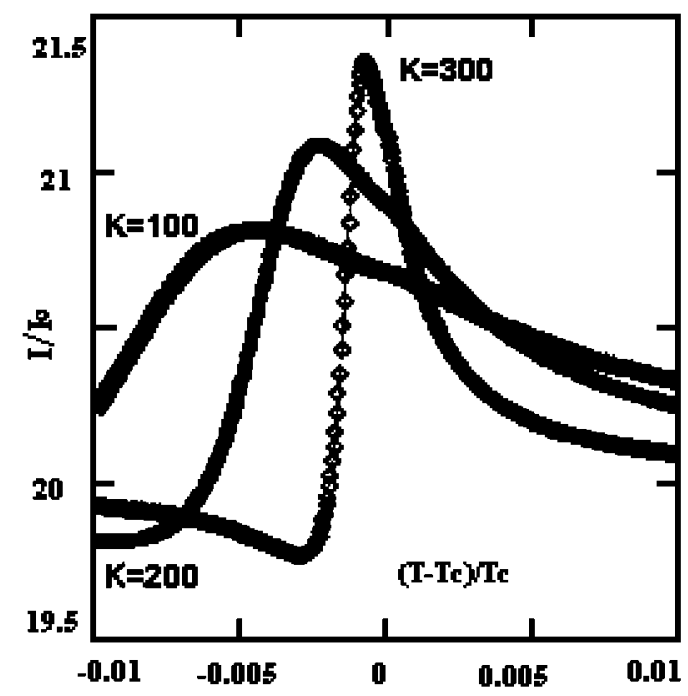

Figure 5. Temperature dependence of the light scattering intensity at the small angles in the finite-size liquid system with the plane-parallel geometry.

It is easy to see, that for $h \rightarrow \infty$ this result is in agreement with the formula which define intensity of scattering light in a spatially infinite system, which follows from main predictions of the OZ theory $I_{\mathrm{OZ}}(k)=I(k \rightarrow 0) /\left(1+R_{\mathrm{c}}^{2} k^{2}\right)$.

From the formula (6.2) follows, that for zero scattering angles and for the critical temperature of volumetric (spatially unlimited) phase, i.e. for $T=T_{\mathrm{c}}$, when $\kappa \rightarrow 0$, the intensity of scattering has no singularity, and it appears proportional to a power of cube of the characteristic size of the system (to an element of dispersing volume) in the direction of space limitlessness (in the considered case - cube of a thickness of the stratum): $I_{1}(0,0,0) \sim\left(16 / \pi^{3}\right) h^{3}$. From the formula (6.2) follows, that in the direction of very small scattering angles $\theta_{x y} \approx 0$ associated with the bounding surface. In the experiment, the anomalous growth of integral intensity of light scattering (critical opalescence) should be observed when the $\left(R_{\mathrm{c} 0}^{-2} \tau^{2 \nu}+\pi^{2} / 4 h^{2}\right)$ becomes equivalent to zero, i.e. not for the bulk critical temperature $T=T_{\mathrm{c}}$. This fact gives an opportunity to define experimentally the new critical temperature for a spatially limited plane-parallel system from the maximum in temperature dependence of light intensity scattering. Figure 5 demonstrates the dependence of the light scattering intensity on temperature variable $\tau$ for the plane-parallel stratum with the thickness equal to $100 R_{\mathrm{c} 0}, 150 R_{\mathrm{c} 0}, 200 R_{\mathrm{c} 0}$, with a corresponding geometrical factor $K=2 h / R_{\mathrm{c} 0}$ is equal to $100,150,200$ and for very small scattering angles $\theta_{x y}, \theta_{z} \approx 10^{-3}$.

Using the similar way as above for a plane-parallel stratum we can now study another spatially limited system, which has got a geometry of cylinder. Taking into account the main contribution to the pair correlation function of a system with a cylindrical geometry at zeroth boundary condition, given by the formula

$$
G_{2}(\rho, z)=D_{1} J_{0}\left(\mu_{1} \rho / a\right) \exp \left[-\sqrt{\kappa_{0}^{2} \tau^{2 \nu}+\mu_{1}^{2} / a^{2}}|z|\right]
$$




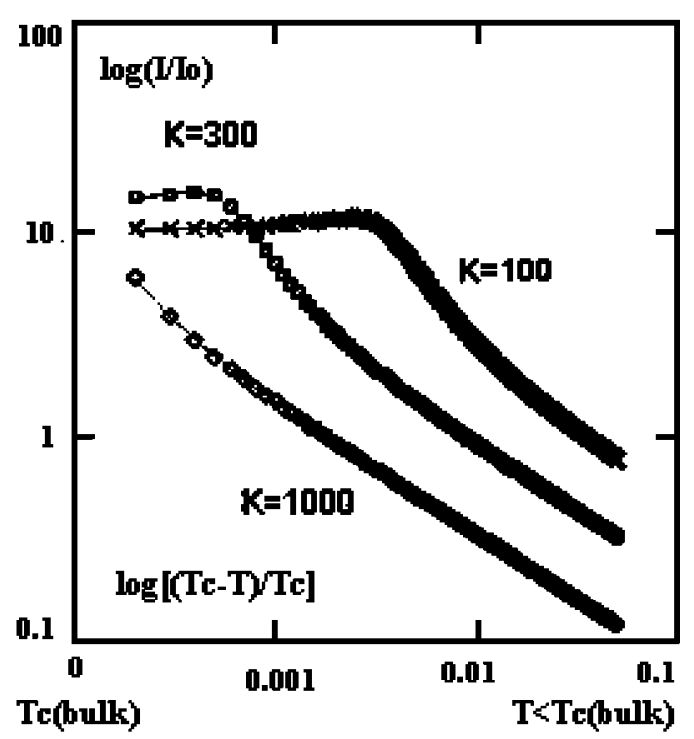

Figure 6. Temperature dependence of the light scattering intensity at the small angles in log-log scale for the finite-size liquid system with a cylindrical geometry.

and executing an integration, one can get

$$
\begin{aligned}
& I_{1}\left(\tau, k_{x y}, k_{z}\right) \sim \\
& \quad \sim \frac{J_{1}\left(\mu_{1}\right)}{\sqrt{a^{2} \kappa_{0}^{2} \tau^{2 \nu}+\mu_{1}^{2}}} \frac{1}{\left(1+\left(a^{2} / 2\right)\left[\left(1-4 / \mu_{1}^{2}\right) k_{x y}^{2}+\left(a^{2} \kappa_{0}^{2} \tau^{2 \nu}+\mu_{1}^{2}\right) k_{z}^{2}\right]\right)},
\end{aligned}
$$

where $J_{0}$ - cylindrical function of zero order, $\mu_{1}=2.4048$ is the first naught of the zero order cylindrical function. From this formula follows in particular, that the intensity of the light scattering remains final even for $\tau=0$, i.e. for the critical temperature of the volumetric phase. Figure 6 demonstrates the dependence of the light scattering intensity on the temperature variable $\tau$ with geometrical factor $K=$ $a / R_{\mathrm{c} 0}$ is equal to $100,300,1000$. When reaching the new critical temperature $T_{\mathrm{c}}^{*}(a)$ of the liquid with a cylindrical form, the expression which defines component intensities in the direction of z-axes has a the following form:

$$
\left(I_{1}\right)_{z} \sim\left[\operatorname{Re} \int \exp \left(-\mathrm{i} k_{z} z\right) \exp \left(-\sqrt{\kappa_{0}^{2} \tau^{* 2 \nu}+\mu_{1}^{2} / a^{2}} z\right) \mathrm{d} z\right]\left[\kappa_{0} \tau^{* \nu} /\left(\kappa_{0}^{2} \tau^{* 2 \nu}+k_{z}^{2}\right)\right] .
$$

From this follows, that at $k_{z} \rightarrow 0$ and $\tau^{*} \rightarrow 0$ the intensity of scattered light increases unlimitedly. This conclusion, as well as in the case of the plane-parallel stratum, could be explained for the direction of limitlessness (along z-axes for the cylinder or in the planes which are parallel to the boundary surface for the stratum) the correlation of the density fluctuations ceases decreasing exponentially at the new critical temperature $T_{\mathrm{c}}^{*}$ and, as a corollary, only in these directions in the experiment a divergence of a correlation length and critical opalescence should be observed. In the above considered examples, the limited systems have the final sizes in fact only in one (for the stratum) or two (for the cylinder) directions. The availability of limitlessness even in one direction is the strongly requested condition for realization 
of a defining indication of the critical condition - the divergence of the correlation length. However, in the system having the form of a sphere, i.e. a comprehensively limited system, the mentioned condition cannot be satisfied. The correlation length under such a geometry remains constant and could reach its maximum value, equal to the sphere's radius $s$. Therefore in such systems the critical state (in its usual understanding) is absent. Accordingly, the critical state's most characteristic manifestations - critical opalescence of light - should not be observed.

\section{Conclusions}

As it is shown, the Munster iteration procedure may be applied to the wide class of the systems: spatially infinite and finite-sized, one-component liquids and binary mixtures, isotropic systems and the systems having anisotropy. It allows us to calculate the nonsingular expressions for the pair correlation functions of the order parameter fluctuations, the correlation length and the shifts of the critical parameters. The abovementioned systems have a great practical application and, therefore, theoretical results received in the paper and especially the results of the critical light scattering might be used for the discussion of the corresponding experimental data.

\section{References}

1. Yukhnovskii I.R. Phase Transitions of the Second Order. The Collective Variables Method. Kiev, Nauk. Dumka, 1985 (in Russian).

2. Patashinskii A.Z., Pokrovskii V.L. Fluctuation Theory of Phase Transition. Oxford, Pergamon Press, 1979.

3. Ornstein L.S., Zernike F. // Proc. Roy. Acad. Amsterdam, 1914, No. 17, p. 793-806.

4. Munster A. The theory of fluctuations. - In: Proceedings of the Enrico Fermi International School of Physics. New York, 1959.

5. Vasil'ev A.N. Chalyi A.V. // Ukr. Phys. J., 1998, No. 5, vol. 43, p. 572-576 (in Ukrainian).

6. Croxton C.A. Liquid State Physics. Cambridge University Press, 1974.

7. Stenley H. Introduction To Phase Transitions And Critical Phenomena. Clarendon Press, Oxford, 1971.

8. Chalyi A.V., Chernenko L.M. Phase transitions in finite-size systems and synaptic transmission. - In: Dynamical Phenomena at Interfaces, Surfaces and Membranes. New York, 1993, p. 457-464.

9. Chalyi A.V. // J. Mol. Liquids, 1993, vol. 58, p. 179-195.

10. Lutz H., Gunton J.D. et al. // Sol. State Comm. 1974, No. 14, p. 1075-1078.

11. Vasil'ev A.N., Chalyi A.V. // Ukr. Phys. J., 2000, No. 1, vol. 45, p. 118-123 (in Ukrainian). 


\title{
Критична поведінка обмежених систем
}

\author{
О.В.Чалий ${ }^{1}$, К.О.Чалий ${ }^{2}$, Л.М.Черненко ${ }^{3}$,
} О.М.Васильєв ${ }^{4}$

1 Кафедра фізики, Національний медичний університет, 01601 Київ, бульв. Шевченка, 13

2 Факультет біо. та хім. інженерії, Університет Ганма, Кіру 376-8515, Японія

3 Інститут хімії поверхні НАН України, 252028 Київ, просп. Науки, 31

4 Фізичний факультет,

Київський національний університет ім. Т.Шевченка, 03022 Київ, просп. Глушкова, 6

Отримано 23 лютого 2000 р.

Досліджено критичні явища та особливості фазових переходів в обмежених рідких системах. Для з'ясування характеру впливу просторової обмеженості на критичні характеристики рідини обрана система з геометрією плоского паралельного прошарку. 3 метою знаходження парних та прямих кореляційних функцій було використано ідеї ітераційного методу Мюнстера. Отримано вираз для радіуса кореляції флуктуацій параметра порядку і відповідні результати проаналізовані в термінах гіпотези подібності. Окрему увагу приділено розрахунку зсуву критичних параметрів (температури та густини). Для дослідження анізотропних систем використано тримоментне наближення. Аналіз кореляційних властивостей бінарних рідких сумішей проводився з використанням системи інтегральних рівнянь Орнштейна-Церніке (ОЦ). Показано, що для рідин з ізоморфним характером міжмолекулярної взаємодії може бути використане наближення, яке спрощує задачу до моделі однокомпонентної рідини. Знайдено асимптотичні формули для парних кореляційних функцій і показано принципову можливість застосування методу Мюнстера для розгляду бінарних сумішей. Розглянуто особливості критичної опалесценції світла для систем зі спеціальною геометрією.

Ключові слова: критичні явища, фазові переходи, обмежена система, кореляційна функція

PACS: 05.70.Fh, 05.70.Jk 